\title{
Bongkrekic Acid as a Warburg Effect Modulator in Long-term Estradiol-deprived MCF-7 Breast Cancer Cells
}

\author{
SHUSO TAKEDA ${ }^{1,2}$, HIROYUKI OKAZAKI ${ }^{2}$, TAICHI KUDO ${ }^{2}$, KAZUHIRO KAKIZOE ${ }^{2}$, \\ TAICHI HIMENO ${ }^{2}$, KENJI MATSUMOTO ${ }^{3}$, MITSURU SHINDO ${ }^{3}$ and HIRONORI ARAMAKI ${ }^{2,4}$ \\ ${ }^{1}$ Laboratory of Xenobiotic Metabolism and Environmental Toxicology, \\ Faculty of Pharmaceutical Sciences, Hiroshima International University (HIU), Hiroshima, Japan; \\ ${ }^{2}$ Department of Molecular Biology, Daiichi University of Pharmacy, Fukuoka, Japan; \\ ${ }^{3}$ Institute for Materials Chemistry and Engineering, Kyushu University, Kasuga, Japan; \\ ${ }^{4}$ Drug Innovation Research Center, Daiichi University of Pharmacy, Fukuoka, Japan
}

\begin{abstract}
Background/Aim: An in vitro cell model of long-term estrogen-deprived MCF-7 (LTED) cells has been utilized to analyze the re-growth mechanisms of breast cancers treated with blockers for estrogen receptor $\alpha$ (ER $\alpha)$ signaling. Bongkrekic acid (BKA) is a natural toxin isolated from coconut tempeh contaminated with the bacterium Burkholderia cocovenans. Materials and Methods: LTED cells, MCF-7 cells and MDA-MB-231 cells were employed in the study. After treatment with BKA (chemically synthesized; purity: $>98 \%$ ), several biochemical analyses were carried out. Results: LTED cells were categorized into an oxidative phenotype. When LTED cells were treated with BKA, lactate dehydrogenase A ( $L D H$ A)/pyruvate dehydrogenase kinase 4 (PDK4) were downregulated, thereby prompting the aggressive use of glucose via mitochondrial oxidative phosphorylation and induction of cell death responses. These effects of BKA were not observed in the other breast cancer cells analyzed. Conclusion: We suggest the potential of BKA as an experimental tool for the analysis of cancer biology in LTED cells.
\end{abstract}

Approximately $70 \%$ of breast cancers express estrogen receptor $\alpha(E R \alpha, E S R 1)$ and are primarily subjected to hormone therapy. Approximately, one-third of breast cancer patients develop recurrence; however, the exact mechanism(s) responsible for the recurrence of breast cancer have not yet been established even though a number of mechanisms have

Correspondence to: Hironori Aramaki, Ph.D., Department of Molecular Biology, Drug Innovation Research Center, Daiichi University of Pharmacy, 22-1 Tamagawa-cho, Minami-ku, Fukuoka 815-8511, Japan. Tel: +81 925410161, Fax: +81 925535698, e-mail: haramaki@daiichi-cps.ac.jp

Key Words: Bongkrekic acid, LTED cells, LDH-A, PDK4, BKA, NADH, MTS assay. been suggested (1-3). Blockers of $17 \beta$-estradiol (E2)/ER $\alpha$ signaling, such as tamoxifen, an ER $\alpha$ antagonist, and inhibitors of aromatase (cytochrome P450 19A1), an enzyme that catalyzes the reaction from testosterone to E2, are utilized in hormone therapy to reduce E2 levels (4). Surgical approaches, such as removal of ovaries in premenopausal women, are also effective; however, recurrence has been reported following tumor regression with reduced E2 levels. An in vitro cell model of long-term estrogen-deprived MCF7 (LTED) cells has been established and utilized to investigate the regrowth mechanisms of breast cancers (1-3). Although several pathways have been suggested, including the up-regulation of insulin-like growth factor-1 (IGF-1)mediated signaling (3), further research is required in order to obtain insight into the development of effective modalities to treat breast tumors.

Epidemiological experiments have indicated that the risk of developing breast cancer is higher in women who consume high-fat diets than in those who consume low-fat diets and fatty acid (FA) compositions have been suggested as key determinants for the outcome of breast cancer (5). $\omega$ 6 polyunsaturated FAs, such as linoleic acid (LA, C18:2) and arachidonic acid (C20:4), are known to exert strong tumorstimulating effects, while saturated FAs, such as palmitic acid (PA, C16:0), have inhibitory effects on breast cancer cells $(6,7)$. The mechanisms by which these FAs modulate the proliferation of breast cancer cells remain currently unknown. However, a previous study reported that breast cancer cells overexpress FA-metabolizing enzymes, such as 15-lipoxygenase (15-LOX) and also that FAs may be metabolized by $15-\mathrm{LOX} / \mathrm{Cu}^{2+}$-mediated oxidation, resulting in the overstimulation of breast cancer cell proliferation (7).

Bongkrekic acid (BKA), a poisonous antibiotic (a natural toxin), was first isolated from coconut tempeh contaminated with the bacterium Pseudomonas cocovenans. The definition of a FA is a carboxylic acid (-COOH) with a long hydrocarbon 
chain; when the structure of BKA was examined, the molecule was revealed to be a branched polyunsaturated long chain with a tricarboxylic acid (Figure 1) and resembled the structures of polyunsaturated FAs, such as LA and AA. BKA was initially established as an inhibitory molecule for mitochondrial adenine nucleotide translocase (ANT), also known as the ADP/ATP translocator, a component of the mitochondrial permeability transition pore (MPTP), and used as an abrogator of apoptotic cell death (8-10) in some settings. We recently demonstrated that BKA is a highly selective stimulator of peroxisome proliferator-activated receptor $\gamma$ (PPAR $\gamma)$ activity (11); however, other potential biological activities of BKA remain currently unknown.

The human breast cancer cell lines, ER $\alpha$-negative MDAMB-231 and -positive MCF-7 have been used to study the Warburg effect because they have glycolytic (i.e., aerobic glycolysis) and oxidative (i.e., mitochondrial oxidative phosphorylation; OXPHOS) phenotypes, respectively, for the production of energy for cellular processes $(12,13)$. Since BKA was shown to be "resistant" to $15-\mathrm{LOX}$ and $\mathrm{Cu}^{2+}$ mediated oxidation, in contrast to LA and AA $(11,14,15)$, and because rosiglitazone, an agonist of PPAR $\gamma$, attenuates IGF-1 receptor-mediated survival signaling in vitro and in vivo $(16,17)$, BKA has potential as an agent for the treatment of LTED cells. BKA may be obtained from commercial sources; however, its purity is generally low (approximately 90-95\%). We originally established synthetic methods for BKA with high purity $(>98 \%)$ (18). In the present study, we investigated whether BKA negatively affects the viability of LTED cells by comparing its effects on other breast cancer cell lines, namely, MCF-7 cells (i.e., the parental cells of LTED cells) and MDA-MB-231 cells. The results obtained showed that (i) BKA specifically induced cell death responses in LTED cells and (ii) BKA selectively decreased pyruvate dehydrogenase kinase 4 (PDK4) and lactate dehydrogenase A (LDH-A) levels in LTED cells, thereby prompting these cells to use glucose as an energy source through OXPHOS. Collectively, these results indicate that BKA has potential as a modifier of the Warburg effect in LTED cells.

\section{Materials and Methods}

Reagents. BKA and BKA derivatives (BKA-2 and BKA-3) were synthesized according to established methods $(18,19)$. BKA-1' and BKA-4 were newly synthesized according to previously described methods $(18,19)$. The purities of these compounds were found to be $>98 \%$ by high-performance liquid chromatography (HPLC) and column chromatography. AA, LA and PA were purchased from Cayman Chemical (Ann Arbor, MI, USA) (purity: >98\%). 2Hydroxy PA (2-OH PA) was obtained from Tokyo Chemical Industry (Tokyo, Japan) (purity: >97\%). All other reagents were of analytical grade, commercially available and used without further purification.
Cell cultures. Cell culture conditions and methods were performed as described previously $(20,21)$. Briefly, the human breast cancer cell lines, MDA-MB-231 and MCF-7 (obtained from the American Type Culture Collection, Rockville, MD, USA) were routinely grown in phenol red-containing minimum essential medium $\alpha$ $(\mathrm{MEM} \alpha)$ (Invitrogen, Carlsbad, CA, USA) supplemented with 10 mM HEPES (4-(2-hydroxyethyl)-1-piperazineethanesulfonic acid), $5 \%$ fetal bovine serum, $100 \mathrm{U} / \mathrm{ml}$ of penicillin and $100 \mu \mathrm{g} / \mathrm{ml}$ of streptomycin in a humidified incubator with an atmosphere of 5\% $\mathrm{CO}_{2}$ at $37^{\circ} \mathrm{C}$. Prior to the chemical treatments, medium was changed to phenol red-free MEM $\alpha$ supplemented with $10 \mathrm{mM}$ HEPES, 5\% fetal bovine serum, $100 \mathrm{U} / \mathrm{ml}$ of penicillin and $100 \mu \mathrm{g} / \mathrm{ml}$ of streptomycin (DCC-medium). In order to establish LTED cells using parent MCF-7 cells, LTED cells were derived from MCF-7 cells grown for 6 months in E2-depleted medium (i.e., DCC-medium) based on a previous study (1). Cultures of approximately subconfluence (close to $60 \%$ confluence) in a $100-\mathrm{mm}$ Petri dish were used to seed for experiments on MTS, cell viability, mRNA expression and a transfection analysis (dual-luciferase assay).

Formazan formation analysis (MTS assay). In the MTS assay, cells were seeded on 96 -well plates at a density of $5 \times 10^{3}$ cells/well and FAs and BKA (individual concentrations were indicated in the Figures) were introduced $4 \mathrm{~h}$ after plating. After a 48 -h incubation, cell viability was analyzed using the CellTiter $96{ }^{\circledR}$ Aqueous One Solution Cell Proliferation Assay (MTS reagent; Promega, Madison, WI, USA), according to the manufacturer's instructions. Test chemicals were prepared in appropriate organic solvents, including dimethyl sulfoxide (DMSO) or ethanol. Control incubations contained equivalent additions of solvents with no measurable influence of vehicle on the formation of formazan at the final concentrations used.

Live/dead cell analysis. MCF-7 and LTED cells were seeded on 96well plates at densities of $5 \times 10^{3}$ cells in $200 \mu \mathrm{l}$ cell culture medium and $\operatorname{BKA}(0.01,1$ and $25 \mu \mathrm{M})$ was introduced $4 \mathrm{~h}$ after seeding. After a 48-h incubation, live and necrotic cells were analyzed using Live/Dead Cell Staining Kit II (PromoKine, Heidelberg, Germany), according to the manufacturer's instructions. The fluorescence of calcein-AM and ethidium homodimer III (EthD-III) was measured using the GloMax-Multi Detection System (Promega). In the morphological examination of LTED cells, images were obtained using a Leica DMIL inverted microscope (Leica Microsystems, Wetzlar, Germany) and captured with a Pixera ${ }^{\circledR}$ Penguin $600 \mathrm{CL}$ Cooled CCD digital camera (Pixera Co., Los Gatos, CA, USA). Data were processed using Pixera Viewfinder 3.0 software (Pixera Co.). Breast cancer cells were plated on 6-well plates. Three areas with approximately equal cell densities were identified in each well and images of each of these areas were captured.

Analysis of $L D H-A$ levels by a reverse transcription polymerase chain reaction $(R T-P C R)$. Total RNA was prepared from MDA-MB-231, MCF-7 and LTED cells using the RNeasy kit (Qiagen, Inc., Hilden, Germany) and purified with RNeasy/QIAamp columns (Qiagen, Inc.) and cDNA (cDNA) synthesis, RT and PCR were subsequently performed using the SuperScript One-Step RT-PCR System with Platinum Taq polymerase (Invitrogen). The primers used were as follows: $L D H-A$ (sense), 5'-GCA GCC TTT TCC TTA GAA CAC-3'; $L D H$-A (antisense), 5'-AAT CCA GAT TGC AAC CGC TTC-3'. Primers for the PCR of $\beta$-actin $(A C T B)$ were taken from a previous study (21). PCR was performed under conditions producing template 


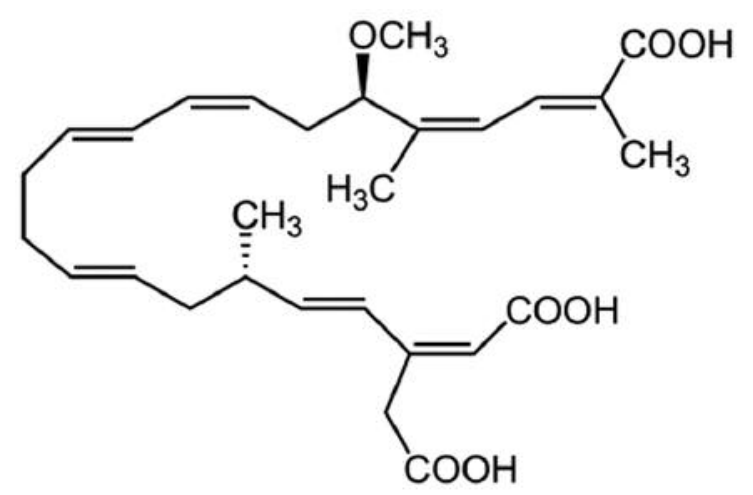

Bongkrekic acid (BKA)

Figure 1. Chemical structure of BKA. BKA has a branched polyunsaturated long chain with a tricarboxylic acid and resembles the structures of polyunsaturated fatty acids.

quantity-dependent amplification over 40 cycles. PCR products were separated by $1.5 \%$ agarose gel electrophoresis in Tris-acetate EDTA buffer and stained with ethidium bromide. When the RT reaction was omitted, no signal was detected in any of the samples. $A C T B$ was used as an internal control for RT-PCR. The quantification of band intensities was performed using ImageJ 1.46r software (http://imagej.nih.gov/ij/).

Real-time RT-PCR analysis. Total RNA was prepared from MDAMB-231, MCF-7 and LTED cells using the RNeasy kit (Qiagen, Inc.) and purified using RNeasy/QIAamp columns (Qiagen, Inc.). In the real-time RT-PCR analysis of $L D H-A, P D K 4$, Topo II $\alpha, K i$ 67, $C P T 1 A$ and $A C T B$, cDNA was prepared via the RT of total RNA using the ReverTra Ace ${ }^{\circledR}$ qPCR RT kit (Toyobo Co. Ltd., Osaka, Japan). Real-time quantitative RT-PCR assays were performed with FastStart Essential DNA Green Master (Roche Applied Science, Indianapolis, IN, USA) and LightCycler ${ }^{\circledR}$ Nano (Roche Diagnostics, Mannheim, Germany). The primers used were as follows; $L D H-A$ (sense), 5'-GCA GCC TTT TCC TTA GAA CAC-3'; $L D H$-A (antisense), 5'-AAT CCA GAT TGC AAC CGC TTC-3'; PDK4 (sense), 5'-AGA GCC TGA TGG ATT TGG TG-3'; $P D K 4$ (antisense), 5'-GCT TGG GTT TCC TGT CTG TG-3'; Topo II $\alpha$ (sense), 5'-CTA TTG AAG AAC TGG CTC CAA-3'; Topo II $\alpha$ (antisense), 5'-CTT TAA ACA GCC TAC GTG GTC-3'; Ki-67 (sense), 5'-TAT CCA GCT TCC TGT TGT GTC-3'; CPT1A (sense), 5'-TC CAG TTG GCT TAT CGT GGT G-3'; CPT1A (antisense), 5'-CTA ACG AGG GGT CGA TCT TGG-3'. The primers for the PCR of $A C T B$ were taken from a previous study (22). The reaction conditions for all mRNAs were $95^{\circ} \mathrm{C}$ for $10 \mathrm{~min}$, followed by 45 cycles at $95^{\circ} \mathrm{C}$ for $10 \mathrm{~s}, 58^{\circ} \mathrm{C}$ for $10 \mathrm{~s}$ and $72^{\circ} \mathrm{C}$ for $15 \mathrm{~s}$. $L D H-A$ PDK4, Topo II $\alpha, K i-67$ and CPTIA mRNA levels were normalized to the corresponding $A C T B$ mRNA levels.

Transfection and luciferase reporter assay (dual-luciferase assay). The day before transfection, MCF-7 cells were seeded $\left(5 \times 10^{4}\right.$ cells/well) on 24-well plates containing MEM $\alpha$ medium. The transfection of each expression plasmid was performed using Lipofectamine ${ }^{\circledR}$ LTX with PLUS $^{\mathrm{TM}}$ reagent (Invitrogen) according

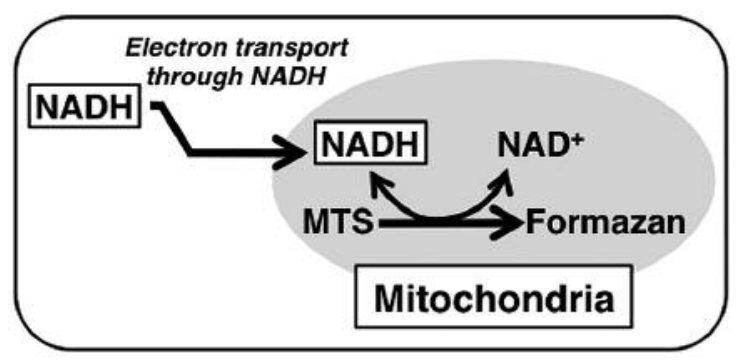

Figure 2. Transportation of cytosolic NADH into mitochondria and principle of the MTS assay. NADH, a reducing equivalent, is produced in the cytosol, followed by transportation into mitochondria. After that, $M T S$ reagent (Owen's reagent) may be reduced by $N A D H$, which results in the formation of a colored formazan product in mitochondria; MTS may be selectively cleaved by mitochondrial succinate dehydrogenase.

to the manufacturer's instructions. The maximal transcriptional efficiencies of the human PPAR $\gamma$ expression plasmid in combination with the human retinoid $\mathrm{X}$ receptor $\alpha(\mathrm{RXR} \alpha)$ plasmid were $100 \mathrm{ng}$ and $100 \mathrm{ng}$, respectively, in the transfections. DNA mixtures of 300 ng of the PPRE-Luc plasmid containing the rat acyl-CoA oxidase PPRE were co-transfected with $20 \mathrm{ng}$ of the Renilla luciferase reporter plasmid (pRL-CMV) in 24-well plates. All plasmid concentrations were equalized with the pcDNA3.1 vector. The expression plasmids of human PPAR $\gamma, \mathrm{RXR} \alpha$ and the PPRE reporter construct were gifts from Dr. Curtis J. Omiecinski (Pennsylvania State University, PA, USA) $(11,23)$. At 24 h post-transfection, cells were washed with phosphate-buffered saline and changed to MEM $\alpha$ without phenol red supplemented with $5 \%$ serum, followed by a treatment with BKA and its derivatives (BKA-1', BKA-2, BKA-3 and BKA-4) for $24 \mathrm{~h}$. After being treated with the compounds, cell extracts were prepared with $100 \mu$ of passive lysis buffer (Promega) and $20 \mu \mathrm{l}$ of the extracts was used for the firefly luciferase and Renilla luciferase assays (Dual-Luciferase Reporter Assay System) by the GloMax-Multi Detection System (Promega). The ratio of firefly luciferase activity (expressed from reporter plasmids) to Renilla luciferase activity (expressed from pRL-CMV) in each sample served as a measure of normalized luciferase activity.

Assay for the formation of conjugated dienes. Experiments were performed based on previously described procedures $(11,15)$. BKA or LA in the presence of copper $\left(\mathrm{Cu}^{2+}, \mathrm{CuSO}_{4}\right)$ was incubated for various times in spectrophotometric cuvettes $(1.0-\mathrm{cm}$ light path) in $100 \mathrm{mM}$ borate buffer at $\mathrm{pH} 9.0$ at room temperature. Absorbance at $234 \mathrm{~nm}$ was periodically recorded after the addition of copper. Results were expressed as an increase in absorbance from the reaction mixture at time zero.

Data analysis. Half-maximal effective concentration $\left(\mathrm{EC}_{50}\right)$ values were obtained using SigmaPlot $11^{\circledR}$ software (Systat Software, Inc., San Jose, CA, USA). Differences were considered significant when the $p$-value was calculated as $<0.05$. Significant differences between two independent groups were calculated by the Student's $t$-test. Other statistical analyses were performed by Scheffé's F test, a posthoc test for analyzing the results of ANOVA. These calculations were performed using Statview 5.0J software (SAS Institute Inc., Cary, NC, USA). 

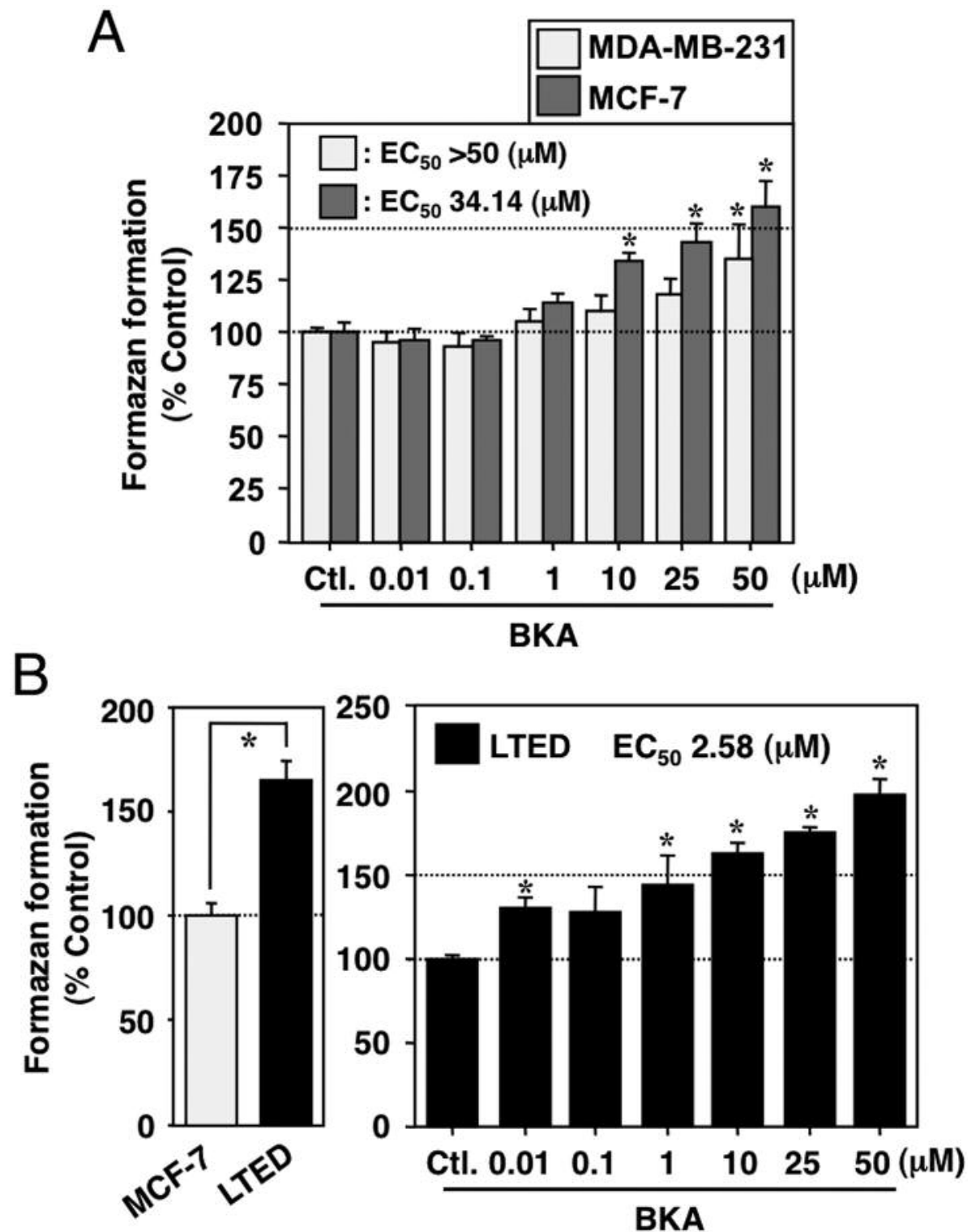

Figure 3. Effects of BKA on formazan formation in three breast cancer cell lines: MDA-MB-231, MCF-7 and LTED cells. (A and B, right panel) Cells were exposed to BKA for $48 \mathrm{~h}$ at concentrations ranging between 0.01 and $50 \mu \mathrm{M}$ to determine $\mathrm{EC}_{50}$ values in formazan formation. After exposures, formazan formation was measured by MTS assay according to the methods described in the Materials and Methods. (B, left panel) Comparison of basal formazan formation between MCF-7 and LTED cells. Cells were used for the MTS assay after seeding for 48 h. Data are expressed as a percentage of the vehicle-treated group (indicated as Ctl.) or MCF-7 cells, as the mean $\pm S E(n=6)$. *Significantly different ( $p<0.05$ ) from the vehicle-treated control or MCF-7 cells.

\section{Results and Discussion}

Effects of BKA on formazan formation in ER $\alpha$-positive (MCF7 and LTED cells) and ER $\alpha$-negative MDA-MB-231 cells. We first performed an MTS assay using a reagent of MTS [3-(4,5dimethylthiazol-2-yl)-5-(3-carboxymethophenyl)-2-(4sulfophenyl)-2H-tetrazolium; Owen's reagent] (24) to analyze whether BKA (Figure 1) and FAs affect the formation of formazan in breast cancer cells. The MTS assay utilizes reducing equivalents, such as the co-enzyme nicotinamide adenine dinucleotide phosphate reduced form (NADH) to convert MTS into a colored formazan product; MTS may be selectively cleaved by mitochondrial succinate dehydrogenase, which is a component of complex II (Figure 2). The MTS assay is generally used as an alternative method for $\left[{ }^{3} \mathrm{H}\right]$ thymidine incorporation. As shown in Figure 3, we investigated the effects 

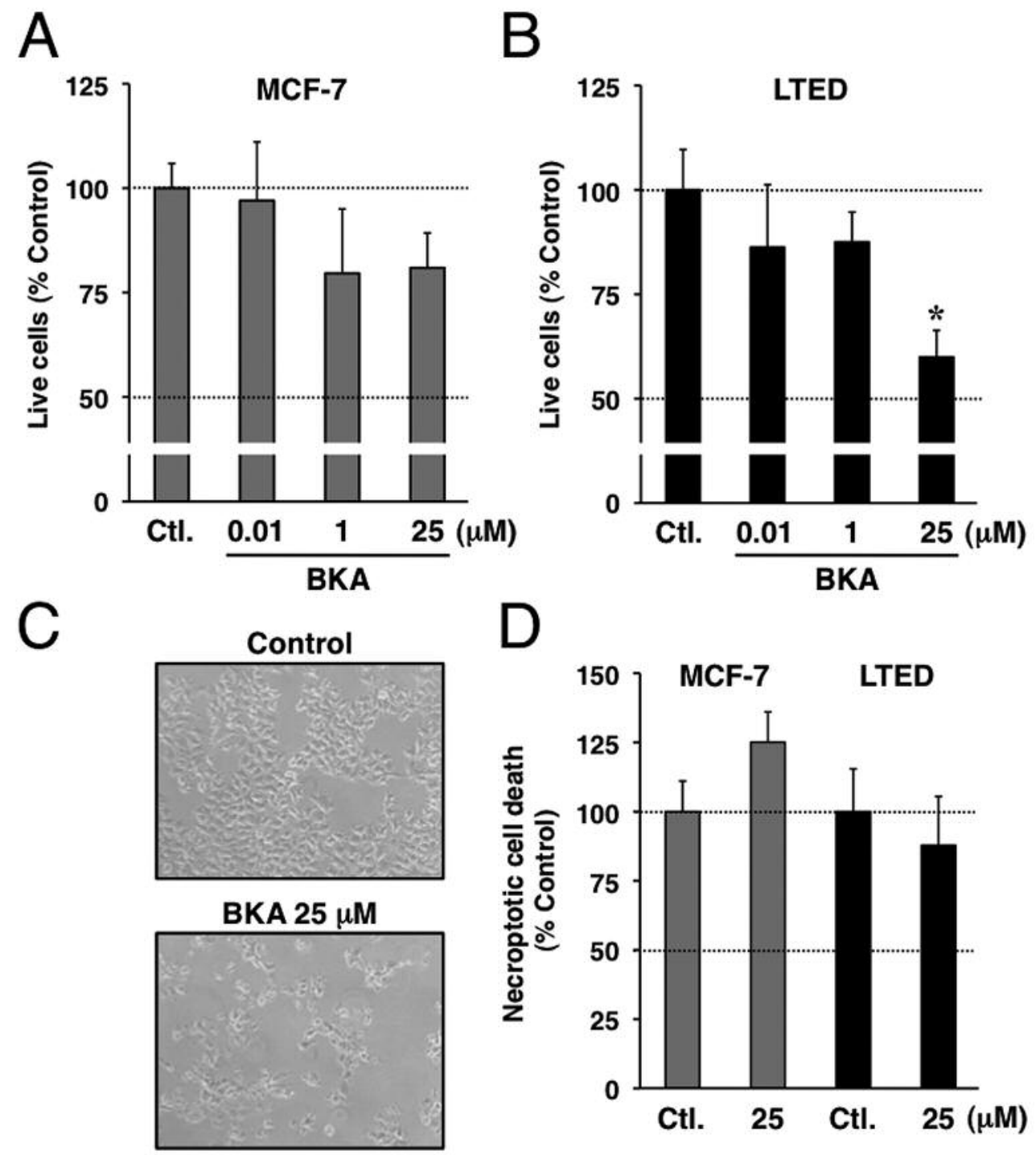

Figure 4. BKA-mediated decreases in living cell numbers: a comparison of LTED cells and parent MCF-7 cells. (A, B and D) MCF-7 and LTED cells were exposed to BKA $(0.01,1$ and $25 \mu M)$ for $48 \mathrm{~h}$. After the treatments, live and necrotic cells were analyzed using calcein-AM and EthD$I I I$, respectively. The fluorescence of calcein-AM/EthD-III was measured by the GloMax-Multi Detection System. (C) LTED cells were treated with vehicle (Ctl.) and $25 \mu \mathrm{MBKA}$ for $48 \mathrm{~h}$ prior to the morphological examination. Representative data images are shown. Images were taken at $\times 200$ magnification. Data are expressed as a percentage of the vehicle-treated group (indicated as Ctl.), as the mean $\pm S E(n=6)$. *Significantly different $(p<0.05)$ from the vehicle-treated control.

of BKA on the formation of formazan in three breast cancer cell lines: MDA-MB-231, MCF-7 and LTED. Although BKA positively enhanced the formation of formazan in MCF-7 cells $\left(\mathrm{EC}_{50}=34.14 \mu \mathrm{M}\right)$, the molecule weakly stimulated its formation in MDA-MB-231 cells with an $\mathrm{EC}_{50}$ value of $>50$ $\mu \mathrm{M}$ (Figure 3A). When MCF-7 cells were compared with LTED cells, the basal formation of formazan was approximately 1.5 -fold higher in LTED cells than in parent MCF-7 cells (Figure 3B, left panel), even though these cells were seeded at the same cell number $\left(5 \times 10^{3}\right.$ cells/well $)$. In the trial with LTED cells, BKA effectively stimulated formazan formation with an $\mathrm{EC}_{50}$ value of $2.58 \mu \mathrm{M}$ (Figure $3 \mathrm{~B}$, right panel). Thus, BKA appears to preferentially accelerate the formation of formazan in breast cancer cells, particularly ER $\alpha$-positive LTED cells.

BKA-mediated decreases in living cells: a comparison of LTED cells and parent MCF-7 cells. The enhanced formation of formazan is generally considered to reflect the number of living cells (i.e., cell viability). If this is the case 
in LTED cells treated with BKA, results using other approaches to examine living cells may be the same or similar to those obtained by the MTS assay. We utilized a calcein (calcein AM) probe and trypan blue dye to detect live cells. This calcein AM probe readily passes through the membranes of "viable cells". After being transported into live cells, calcein is hydrolyzed by cytosolic esterases into a green-fluorescent calcein. Since dead cells lack esterases, live cells are selectively marked with the probe. Although BKA had no observable effects on the viability of MDAMB-231 cells, even at $25 \mu \mathrm{M}$ (data not shown), it slightly reduced the number of live MCF-7 cells at concentrations up to $25 \mu \mathrm{M}$ (Figure $4 \mathrm{~A}$ ) and significantly reduced the number of live LTED cells in a concentration-dependent manner (Figure 4B). The BKA-mediated suppression of cell growth was detected with the trypan blue exclusion test (data not shown). In support, the morphology of LTED cells was affected (implicating cell death responses) more by BKA than vehicle-treated control cells (Figure 4C). Furthermore, an analysis of the cell death type using an EthD-III probe indicated that BKA-mediated death was not dependent on necroptotic cell death (Figure 4D).

Expression status of $L D H-A$ in breast cancer cells and effects of BKA on the expression of LDH-A. LDH-A is one of the major isoforms of LDH expressed in breast tissues that regulates the conversion of pyruvate to lactate in the cellular glycolytic process (25). MDA-MB-231 and MCF-7 breast cancer cell lines have been used to study the Warburg effect $(12,13)$; highly aggressive MDA-MB-231 cells are known to obtain ATP by the glycolytic pathway, even in the presence of $\mathrm{O}_{2}$ (i.e., the "glycolytic" phenotype), which converts glucose to lactate and reduces OXPHOS activity (Figure 5A). In contrast to MDA-MB-231 cells, noninvasive/less tumorigenic MCF-7 cells utilized the OXPHOS pathway to produce ATP with lower glycolytic activity (i.e., the "oxidative" phenotype) (Figure 5B). In support of the above-described phenomena, decreases in LDH-A levels correlated with increases in cytosolic $\mathrm{NADH} / \mathrm{NAD}^{+}$, which is consistent with a higher mitochondrial respiration rate (26) (Figures 5A and 4B). Thus, we focused on the expression status of LDH-A in the three breast cancer cells. Semiquantitative and real-time RT-PCR analyses indicated that $L D H-A$ expression was detected in the following order: MDA-MB-231 cells (5.79-, 2.3-fold) >> MCF-7 cells (1.0fold) $=$ LTED cells $(0.76-, 1.3$-fold) (Figure 6A). Therefore, LTED cells were categorized into the "oxidative phenotype", similar to the parent MCF-7 cells. We then investigated the effects of BKA and FAs (PA and AA) on the expression of LDH-A in breast cancer cells; BKA and FA concentrations were fixed at $25 \mu \mathrm{M}$ based on the results shown in Figure 6. AA did not exert any modulating activity on any of the breast cancer cells examined. In spite of the cell type, PA up-

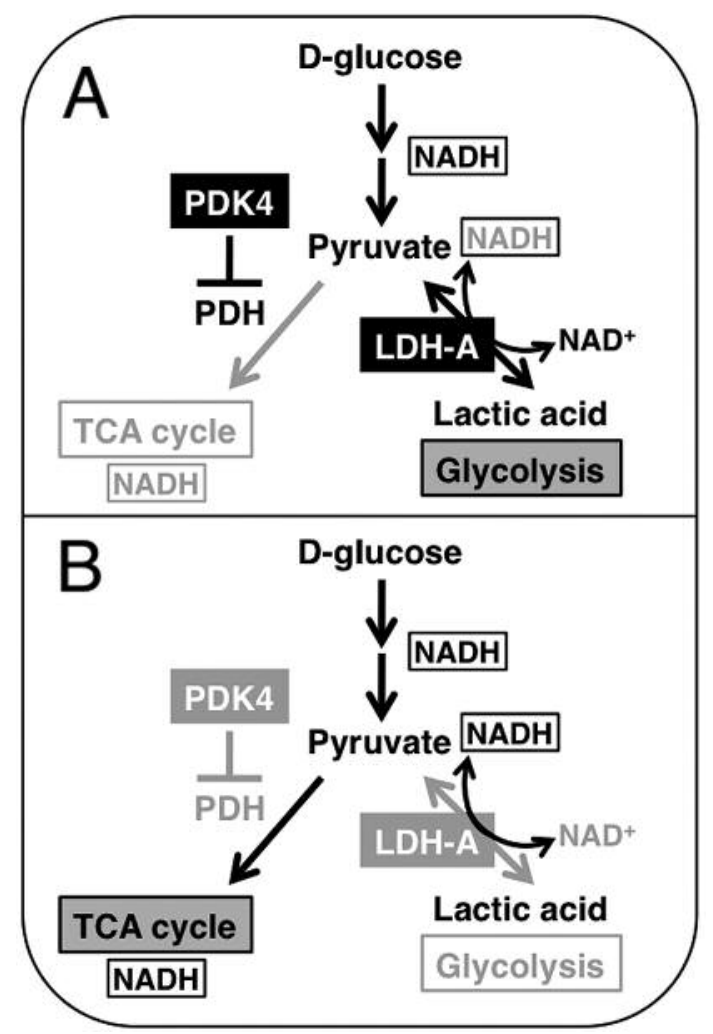

Figure 5. Schematic representation of differences between aerobic glycolysis and oxidative phosphorylation (OXPHOS). (A) Cancer cells, including $M D A-M B-231$ cells, rely on aerobic glycolysis to generate ATP. MDA-MB-231 cells expressed higher levels of LDH-A and PDK4 than MCF-7 and LTED cells. (B) In contrast to MDA-MB-231 cells, $M C F-7$ and LTED cells rely primarily on mitochondrial OXPHOS to generate ATP.

regulated the expression of $L D H-A$, whereas BKA significantly down-regulated its expression in LTED cells (0.66-fold) compared to control (1.0-fold) (Figures 6B-D).

Expression status of PDK4 in breast cancer cells and effects of BKA on the expression of PDK4. Previous studies reported that cellular glucose oxidation is decreased by FAs due to the induction of PDK4, a key enzyme for the regulation of glucose (carbohydrate) oxidation via the inhibition of the pyruvate dehydrogenase complex $(\mathrm{PDH})$ in mitochondria $(27$, 28) (see Figure 5A). Taken together with the results described in Figure 6, BKA appears to modulate the expression status of "key enzymes" responsible for the metabolic fate of glucose. The results of the real-time RT-PCR analysis demonstrated that the basal expression of PDK4 varied markedly among the three breast cancer cells tested; MDA-MB-231 cells (2.18fold) expressed the highest levels of $P D K 4$, followed by MCF7 cells (1.0-fold) and LTED cells (0.08-fold) (Figure 7A). Thus, LTED cell growth appears to be highly dependent on 


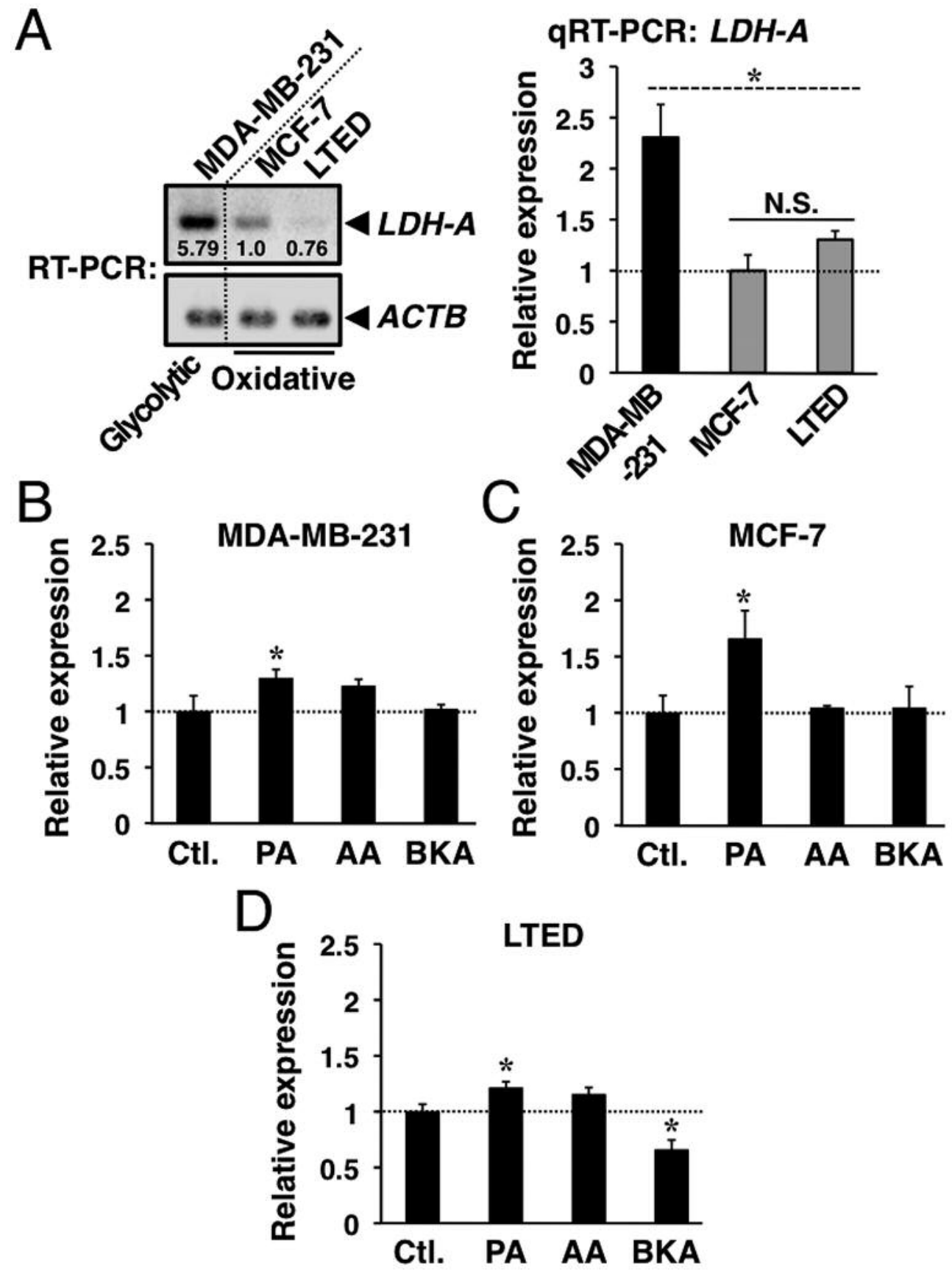

Figure 6. Expression status of LDH-A in breast cancer cells and effects of BKA on the expression of LDH-A. (A) Semi-quantitative RT-PCR (A, left panel) and real-time RT-PCR (A, right panel) analyses of LDH-A basally expressed in individual MDA-MB-231, MCF-7 and LTED cells. Betaactin (ACTB) was used an internal loading control. Band intensities were quantified by densitometry (ImageJ $1.46 \mathrm{r}$ software) and normalized to ACTB levels (A, left panel). (B-D) Real-time RT-PCR analyses of LDH-A in MDA-MB-231, MCF-7 and LTED cells 48 h after the treatment with vehicle (indicated as Ctl.), PA, LA or BKA $(25 \mu \mathrm{M})$. Data are expressed as a fold change or induction from MCF-7 cells or the vehicle-treated control, as the mean \pm S.E. $(n=3)$. *Significantly different $(p<0.05)$ from each cell or the vehicle-treated control. N.S., Not significant.

glucose (i.e., glucose oxidation) as an energy source. We then investigated the effects of $B K A$ and two FAs (PA and AA) on the three breast cancer cells. As expected, the expression of $P D K 4$ was significantly stimulated by the two FAs in MDA-
MB-231 cells. However, AA up-regulated the expression of PDK4, whereas PA down-regulated it in MCF-7/LTED cells (Figures 7B-D). It is important to note that, in contrast to the effects of FAs, BKA "selectively" down-regulated the 

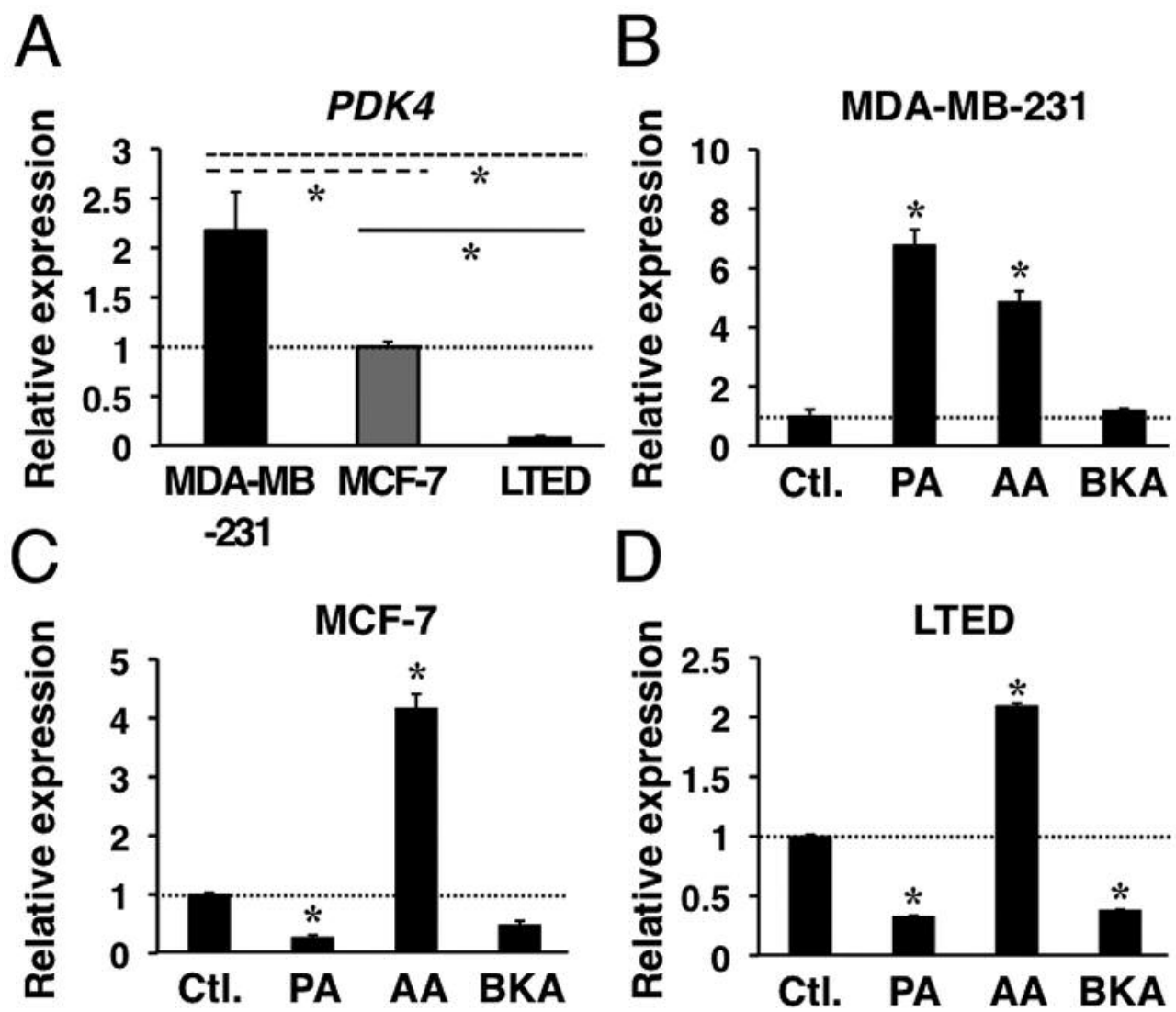

Figure 7. Expression status of PDK4 in breast cancer cells and effects of BKA on the expression of PDK4. (A) Real-time RT-PCR analyses of PDK4 basally expressed in individual MDA-MB-231, MCF-7 and LTED cells. (B-D) Real-time RT-PCR analyses of PDK4 in MDA-MB-231, MCF-7 and LTED cells $48 \mathrm{~h}$ after the treatment with vehicle (indicated as Ctl.), PA, LA or BKA (25 $\mu M)$. Data are expressed as a fold change or induction from $M C F-7$ cells or the vehicle-treated control, as the mean \pm S.E. $(n=3)$. *Significantly different $(p<0.05)$ from each cell or the vehicle-treated control.

expression of PDK4 in LTED cells (Figure 7D) and, as shown in Figure 6D, $L D H-A$ in LTED cells was identified as a target of BKA. Thus, BKA-mediated decreases in PDK4/LDH-A levels may relieve the inhibition of PDH, thereby prompting the aggressive use of glucose.

Expression status of Topo II $\alpha$ in breast cancer cells and effects of BKA on the expression of Topo II $\alpha$. We measured the expression pattern of the proliferation marker Topo II $\alpha$ in the three breast cancer cells. Real-time RT-PCR analyses revealed that the strongest expression of Topo II $\alpha$ was detected in MDA-MB-231 cells (1.3-fold), followed by MCF-7 cells (1.0fold) $>$ LTED cells ( 0.54 -fold). Although the reason why Topo II $\alpha$ was more weakly expressed in LTED cells than in parent MCF-7 cells remains currently unclear, it is speculated that highly aggressive MDA-MB-231 breast cancer cells express the highest levels of Topo II $\alpha$ (Figure 8A). This expression order of Topo II $\alpha$ was similar to that of PDK4 (Figure 7A). Consistent with the results shown in Figure 8B, PA at $25 \mu \mathrm{M}$ decreased the viability of MDA-MB-231 cells (data not shown) (6). When the effects of BKA were investigated in more detail, we found that this molecule down-regulated the expression of Topo II $\alpha$ in LTED cells only; this modulative effect by BKA was only observed for the expression of PDK4 in LTED cells (Figures 8B-D; see also Figures 6 and 7). In order to further support the modulation of Topo II $\alpha$ by BKA in LTED cells, we focused on another proliferation marker of cancer cells, Ki-67 (29). Real-time RT-PCR results for the expression of Ki-67 were similar to those of Topo II $\alpha(0.41 \pm 0.014 v s$. Ct1. $=1.0$, $p<0.05$ ), indicating BKA-selective inhibitory effects on the proliferation of LTED cells. Inversely related to the formation of formazan by BKA (Figure 2B), proliferation markers' (Topo II $\alpha$ and Ki-67) levels and living cell numbers were reduced by BKA in LTED cells (Figures 3B and 8D).

Effects of simplified BKA analogs on formazan formation and PPAR $\gamma$-mediated transcriptional activity. As we reported previously, BKA has been established as a selective activator 

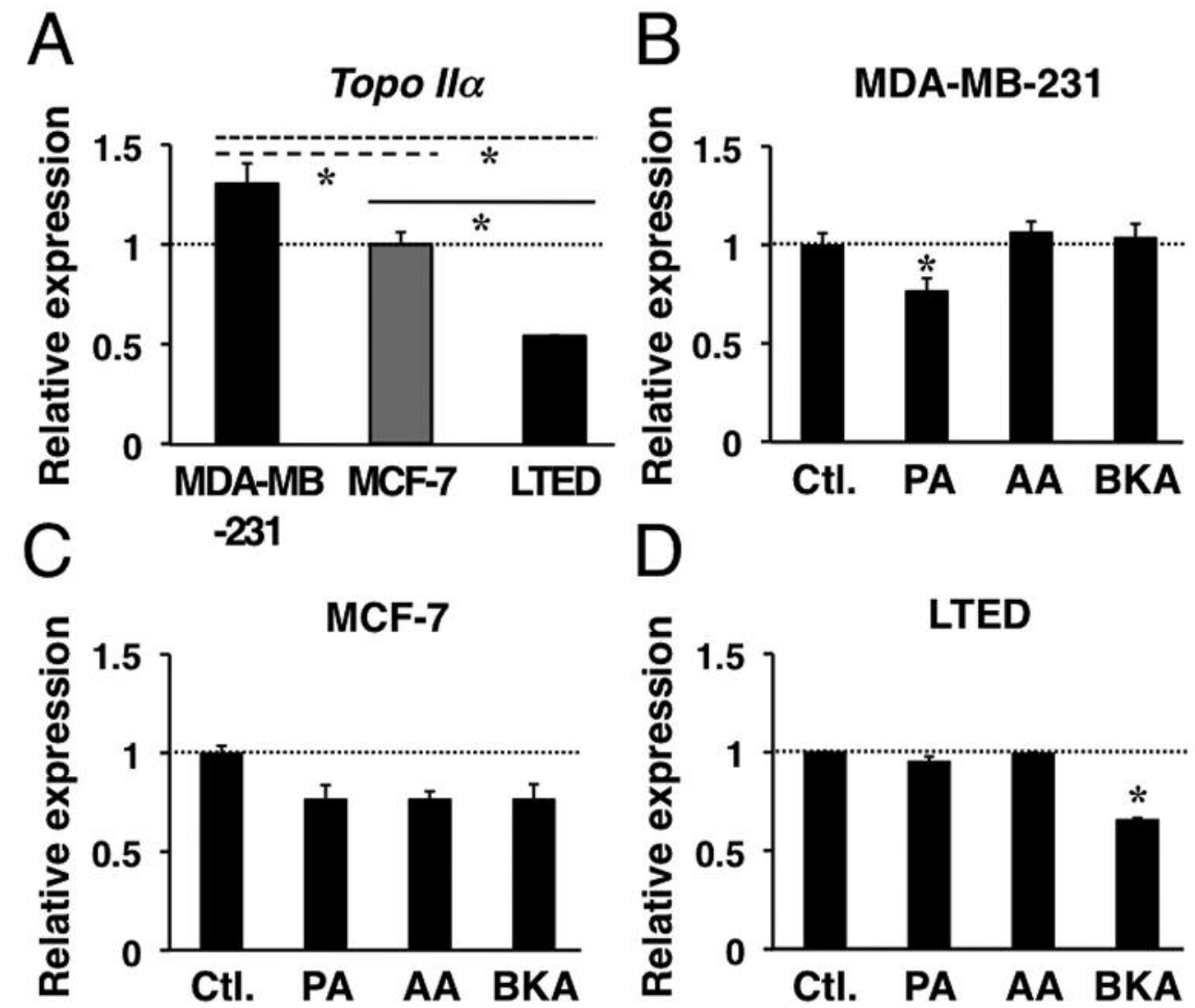

Figure 8. Expression status of Topo II in breast cancer cells and effects of BKA on the expression of Topo IIL. (A) Real-time RT-PCR analyses of Topo II a basally expressed in individual MDA-MB-231, MCF-7 and LTED cells. (B-D) Real-time RT-PCR analyses of Topo II $\alpha$ in MDA-MB-231, MCF-7 and LTED cells $48 \mathrm{~h}$ after the treatment with vehicle (indicated as Ctl.), PA, LA or BKA (25 $\mu M)$. Data are expressed as a fold change or induction from MCF-7 cells or the vehicle-treated control, as the mean $\pm S . E .(n=3)$. *Significantly different $(p<0.05)$ from each cell or the vehicletreated control.

for PPAR $\gamma$, coupled with the up-regulation of its regulated gene, adiponectin $(A D I P O Q)$, but not with fatty acid 2hydroxylase $(F A 2 H)$, a PPAR $\alpha$-regulated gene $(11,23)$. After switching parent MCF-7 cells into LTED cells cultured under reduced E2 conditions, growth signaling mediated by IGF-1 (i.e., mitogenic and anti-apoptotic effects) was observed (30) and, in some settings, the activation of PPAR $\gamma$ by rosiglitazone suppressed the IGF regulatory system in vitro and in vivo (16). Furthermore, a strong positive association has been reported between IGF-1 levels and breast cancer (30). Based on these findings, we hypothesized that the selective anti-proliferative effects of BKA on LTED cells are based on its association with PPAR $\gamma$. As shown in Figure 9A, we originally synthesized two simplified BKA analogs, BKA1 ' and BKA-4, and applied them in addition to BKA, BKA2 and BKA-3 (11) to the formazan formation assay at 10 and $50 \mu \mathrm{M}$. Of these, only BKA-3 exhibited significant, but weaker stimulating activity at $50 \mu \mathrm{M}$ than that of parent BKA (Figures 3B and 9B). Transcriptional experiments on PPAR $\gamma$ revealed that BKA-3 (4.86-fold) and BKA-4 (4.18-fold) in addition to BKA (2.07-fold) activated PPAR $\gamma$ (Figure 9C). Although the PPAR $\gamma$ activation potentials of BKA-3/BKA-4 were approximately 2-fold stronger than that of BKA, they exhibited very weak or negative effects on formazan formation. Thus, the stimulatory effects of BKA on PPAR $\gamma$ are not assertively involved in LTED-directed cell death.

The IGF-1-mediated down-regulation of PDK4 has been reported (31). Thiazolidinediones (rosiglitazone and pioglitazone), which are PPAR $\gamma$ agonists, have been shown to stimulate IGF-1 signaling via a PPAR $\gamma$-independent pathway in human aortic smooth muscle cells (32). Although this cell system is different from that used in the present study, if this signaling plays a role in the effects of BKA, BKA may utilize similar pathway(s) to thiazolidinediones in LTED cells. The capacity to transport cytosolic NADH into mitochondria is reduced in rapidly proliferating cells (33). If this finding is applied to aggressive MDA-MB-231 cells, the transporting systems for NADH in MCF-7 and LTED cells are up-regulated 

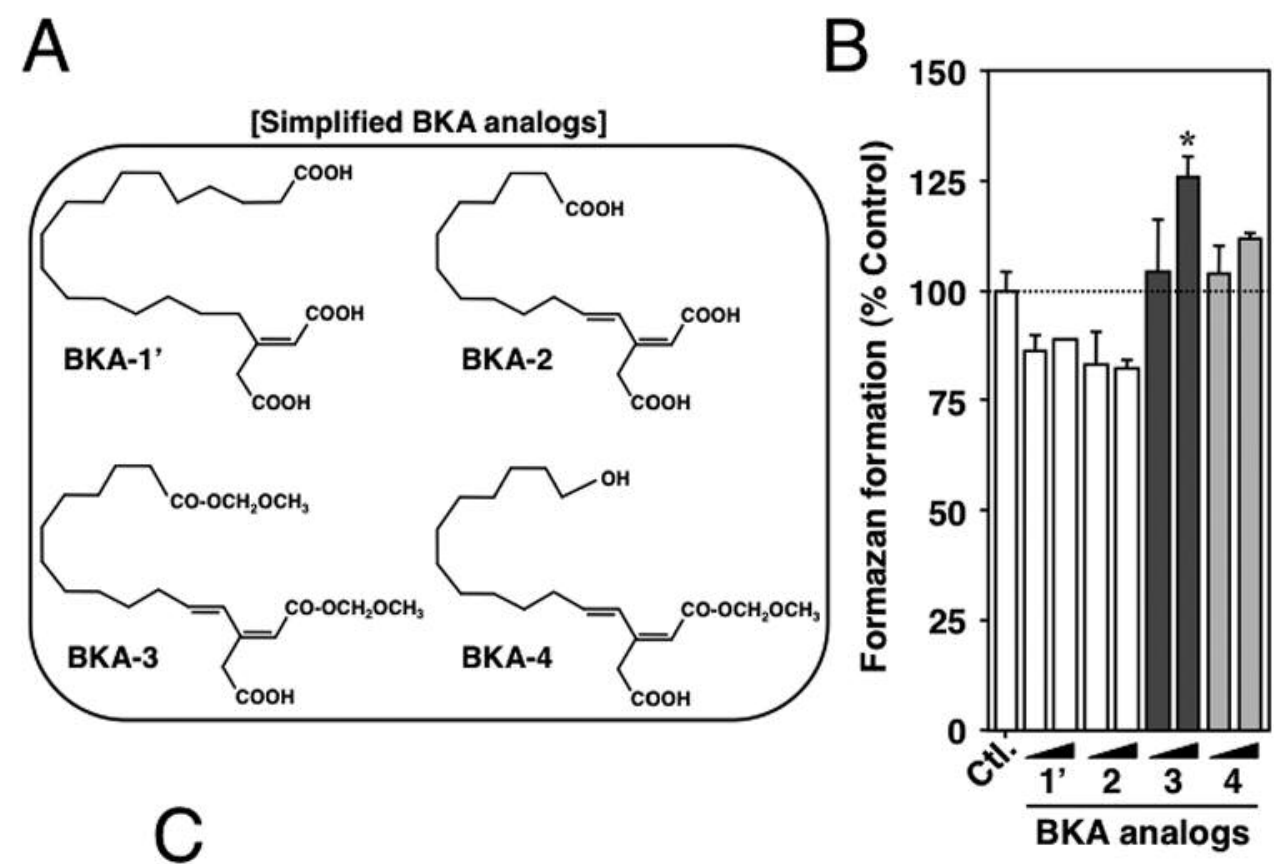

\begin{tabular}{|c|c|c|}
\hline BKA and its analogs & PPAR $\gamma$ activity & Reference \\
\hline BKA & $2.07 \pm 0.059$ & Okazaki et al., 2015 \\
\hline BKA-1' & $1.27 \pm 0.036$ & This work \\
\hline BKA-2 & $1.26 \pm 0.031$ & Okazaki et al., 2015 \\
\hline BKA-3 & $4.86 \pm 0.073$ & Okazaki et al., 2015 \\
\hline BKA-4 & $4.18 \pm 0.098$ & This work \\
\hline
\end{tabular}

Figure 9. Effects of BKA analogs on formazan formation and PPAR -mediated transactivation activity. (A) Chemical structures of BKA analogs (BKA-1', 2, 3 and 4). (B) LTED cells were exposed for $48 \mathrm{~h}$ to BKA (10 and $50 \mu \mathrm{M})$. After the treatments, formazan formation was measured by the MTS assay according to the methods described in the Materials and Methods. Data are expressed as a percentage of the vehicle-treated group (indicated as Ctl.), as the mean $\pm S E(n=6)$. *Significantly different $(p<0.05)$ from the vehicle-treated control. $(C)$ MCF-7 cells were transiently transfected with a PPRE-luciferase reporter plasmid in combination with a pcDNA3.1 plasmid and expression plasmid of human PPAR $\gamma$. After transfection, cells were treated with vehicle (Ctl.) or BKA and its analogs (BKA-1', 2, 3 and 4) at $25 \mu M$. After 24 h, cells were harvested and assayed for luciferase activity and all transfections were normalized for efficiency using the internal Renilla control plasmid. Data are expressed as fold activation from the vehicle-treated control (Ctl.), as the mean $\pm S . E .(n=3)$. The results for BKA, BKA-2 and BKA-3 presented in the Figure were taken from reference 11 .

(Figure 2). Taken together with findings demonstrating that extracellularly added NADH (which enters cells and reaches the OXPHOS apparatus) suppresses the proliferation of MCF-7 cells, coupled with cell death accompanying DNA fragmentation (34-36) and formazan formation (NADH production) as a hallmark of mitochondrial activity, the cell death responses induced by BKA appear to be the result of the "overactivation" of mitochondria (Figures 2, 3B, 4B, 5B and 8).

$B K A$ is insensitive to $\mathrm{Cu}^{2+}{ }_{-}$mediated oxidation. Fatty acids are oxidized by 15 -LOX and/or $\mathrm{Cu}^{2+}$-mediated reaction in the body; for example, LA (C18:2) may be metabolized into
$( \pm)$ 13-HpODE (Figure 10A), a positive stimulator for breast cancer cell growth (7). When the structure of BKA was examined, it was found to have a dienylmethylene $(-\mathrm{CH}=\mathrm{CH}-$ $\mathrm{CH}=\mathrm{CH}-\mathrm{CH} 2$-), a possible oxidizable moiety (Figure 1). Thus, we speculated that BKA may be oxidized by $\mathrm{Cu}^{2+}$; however, the results shown in Figure 10B revealed that BKA is insensitive to $\mathrm{Cu}^{2+}$, whereas LA was oxidized. The resistance of BKA to 15-LOX was also demonstrated (data not shown) (11). Furthermore, we did not detect any absorption peaks at wavelengths ranging between $200 \mathrm{~nm}$ and $800 \mathrm{~nm}$ (data not shown). Collectively, these results suggest that BKA is more stable than $\mathrm{LA}$ against $\mathrm{Cu}^{2+}$-mediated oxidation. 

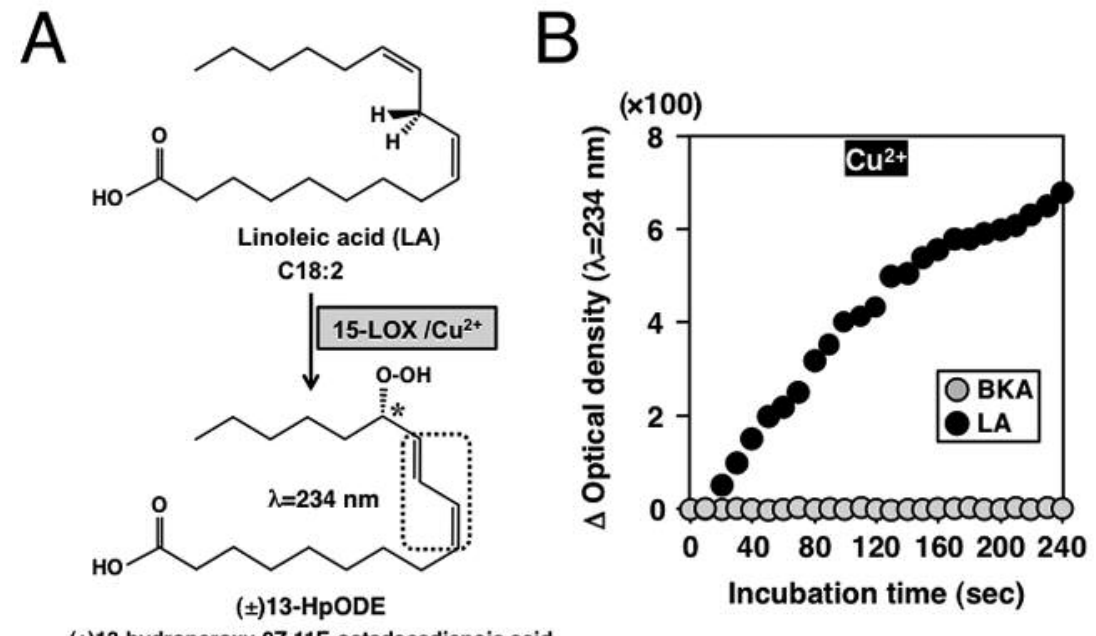

Figure 10. BKA was not sensitive to $\mathrm{Cu}^{2+}$-mediated oxidation. (A) Chemical structure of LA. (B) BKA or LA was incubated with $C u^{2+}$ for up to $240 \mathrm{~s}$ as described in the Materials and Methods. Absorbance at $234 \mathrm{~nm}$ was periodically recorded. Results are expressed as an increase in absorbance from the reaction mixture at time zero. The modified moiety (active methylene) in LA is indicated with an inclusion. ( \pm )13-HpODE, an oxidized metabolite, is also shown.

\section{Conclusion}

In the present study, we did not obtain direct evidence for the interaction between the BKA-induced down-regulation of $L D H$-A/PDK4 (Topo II $/ \mathrm{Ki}$-67) and LTED cell death; however, this is the first study to identify BKA as a highly selective modulator for the metabolic pathway in LTED cells (Figure 5B). BKA has potential as a therapeutic modality for the recurrence of breast cancers that have already been treated with blockers of $17 \beta$-estradiol/ER $\alpha$ signaling; however, further investigations are needed on the mechanism(s) responsible for BKA-mediated cell death in LTED cells.

\section{Conflicts of Interest}

No potential conflicts of interest were disclosed.

\section{Acknowledgements}

This work was performed under the Cooperative Research Program of the Network Joint Research Center for Materials and Devices (Research Nos. 2015454 and 20163071 (to H.A.)). This study was also supported, in part, by JSPS KAKENHI Grant Numbers JP26293004, JP16H01157 (to M.S.).

\section{References}

1 Shim WS, Conaway M, Masamura S, Yue W, Wang JP, Kmar R and Santen RJ: Estradiol hypersensitivity and mitogen-activated protein kinase expression in long-term estrogen deprived human breast cancer cells in vivo. Endocrinology 141: 396-405, 2000.
2 Yue W, Wang JP, Conaway MR, Li Y and Santen RJ: Adaptive hypersensitivity following long-term estrogen deprivation: involvement of multiple signal pathways. J Steroid Biochem Mol Biol 86: 265-274, 2003.

3 Fox EM, Miller TW, Balko JM, Kuba MG, Sánchez V, Smith RA, Liu S, González-Angulo AM, Mills GB, Ye F, Shyr Y, Manning HC, Buck E and Arteaga CL: A kinome-wide screen identifies the insulin/IGF-I receptor pathway as a mechanism of escape from hormone dependence in breast cancer. Cancer Res 71: 6773-6784, 2011.

4 Strasser-Weippl K and Goss PE: Advances in adjuvant hormonal therapy for postmenopausal women. J Clin Oncol 23: 17511759, 2005.

5 Boyd NF, Cousins M, Lockwood G and Tritchler D: The feasibility of testing experimentally the dietary fat-breast cancer hypothesis. Br J Cancer 62: 878-881, 1990.

6 Hardy S, El-Assaad W, Przybytkowski E, Joly E, Prentki M and Langelier Y: Saturated fatty acid-induced apoptosis in MDAMB-231 breast cancer cells. A role for cardiolipin. J Biol Chem 278: 31861-318670, 2003.

7 O'Flaherty JT, Wooten RE, Samuel MP, Thomas MJ, Levine EA, Case LD, Akman SA and Edwards IJ: Fatty acid metabolites in rapidly proliferating breast cancer. PLoS One 8: e63076, 2013.

8 Henderson PJ and Lardy HA: Bongkrekic acid. An inhibitor of the adenine nucleotide translocase of mitochondria. J Biol Chem 245: 1319-1326, 1970.

9 Crompton M: The mitochondrial permeability transition pore and its role in cell death. Biochem J 341: 233-249, 1999.

10 Leung AW and Halestrap AP: Recent progress in elucidating the molecular mechanism of the mitochondrial permeability transition pore. Biochim Biophys Acta 1777: 946-952, 2008.

11 Okazaki H, Takeda S, Ikeda E, Fukunishi Y, Ishii H, Taniguchi A, Tokuyasu M, Himeno T, Kakizoe K, Matsumoto K, Shindo $\mathrm{M}$ and Aramaki H: Bongkrekic acid as a selective activator of 
the peroxisome proliferator-activated receptor $\gamma$ (PPAR $\gamma)$ isoform. J Toxicol Sci 40: 223-233, 2015.

12 Guppy M, Leedman P, Zu X and Russell V: Contribution by different fuels and metabolic pathways to the total ATP turnover of proliferating MCF-7 breast cancer cells. Biochem J 364: 309315,2002

13 Gatenby RA and Gillies RJ: Why do cancers have high aerobic glycolysis? Nat Rev Cancer 4: 891-899, 2004.

14 Takeda S, Usami N, Yamamoto I and Watanabe K: Cannabidiol2',6'-dimethyl ether, a cannabidiol derivative, is a highly potent and selective 15-lipoxygenase inhibitor. Drug Metab Dispos 37: 1733-1737, 2009.

15 Takeda S, Hirayama A, Urata S, Mano N, Fukagawa K, Imamura M, Irii A, Kitajima S, Masuyama T, Nomiyama M, Tatei $S$, Tomita S, Kudo T, Noguchi M, Yamaguchi Y, Okamoto Y, Amamoto T, Fukunishi Y, Watanabe K, Omiecinski CJ and Aramaki H: Cannabidiol-2',6'-dimethyl ether as an effective protector of 15-lipoxygenase-mediated low-density lipoprotein oxidation in vitro. Biol Pharm Bull 34: 1252-1256, 2011.

16 Lecka-Czernik B, Ackert-Bicknell C, Adamo ML, Marmolejos V, Churchill GA, Shockley KR, Reid IR, Grey A and Rosen CJ: Activation of peroxisome proliferator-activated receptor $\gamma$ (PPAR $\gamma$ ) by rosiglitazone suppresses components of the insulinlike growth factor regulatory system in vitro and in vivo. Endocrinology 148: 903-911, 2007.

17 Papageorgiou E, Pitulis N, Manoussakis M, Lembessis P and Koutsilieris M: Rosiglitazone attenuates insulin-like growth factor 1 receptor survival signaling in PC-3 cells. Mol Med 14: 403-411, 2008.

18 Matsumoto K, Suyama M, Fujita S, Moriwaki T, Sato Y, Aso Y, Muroshita S, Matsuo H, Monda K, Okuda K, Abe M, Fukunaga H, Kano A and Shindo M: Efficient total synthesis of bongkrekic acid and apoptosis inhibitory activity of its analogues. Chem Eur J 21: 11590-11602, 2015.

19 Okuda K, Hasui K, Abe M, Matsumoto K and Shindo M: Molecular design, synthesis, and evaluation of novel potent apoptosis inhibitors inspired from bongkrekic acid. Chem Res Toxicol 25: 2253-2260, 2012.

20 Takeda S, Yamamoto I and Watanabe K: Modulation of $\Delta^{9}$ tetrahydrocannabinol-induced MCF-7 breast cancer cell growth by cyclooxygenase and aromatase. Toxicology 259: 25-32, 2009.

21 Takeda S, Yoshida K, Nishimura H, Harada M, Okajima S, Miyoshi H, Okamoto Y, Amamoto T, Watanabe K, Omiecinski $\mathrm{CJ}$ and Aramaki H: $\Delta^{9}$-Tetrahydrocannabinol disrupts estrogensignaling through up-regulation of estrogen receptor $\beta$ (ER $\beta)$. Chem Res Toxicol 26: 1073-1079, 2013.

22 Steuerwald N, Cohen J, Herrera RJ and Brenner CA: Quantification of mRNA in single oocytes and embryos by realtime rapid cycle fluorescence monitored RT-PCR. Mol Hum Reprod 6: 448-453, 2000.

23 Takeda S, Ikeda E, Su S, Harada M, Okazaki H, Yoshioka Y, Nishimura H, Ishii H, Kakizoe K, Taniguchi A, Tokuyasu M, Himeno T, Watanabe K, Omiecinski CJ and Aramaki H: $\Delta^{9}$ THC modulation of fatty acid 2-hydroxylase (FA2H) gene expression: Possible involvement of induced levels of PPAR $\alpha$ in MDA-MB-231 breast cancer cells. Toxicology 326: 18-24, 2014.
24 Cory AH, Owen TC, Barltrop JA and Cory JG: Use of an aqueous soluble tetrazolium/formazan assay for cell growth assays in culture. Cancer Commun 3: 207-212, 1991.

25 Dang $\mathrm{CV}$ and Semenza GL: Oncogenic alterations of metabolism. Trends Biochem Sci 24: 68-72, 1999.

26 Fantin VR, St-Pierre J and Leder P: Attenuation of LDH-A expression uncovers a link between glycolysis, mitochondrial physiology, and tumor maintenance. Cancer Cell 9: 425-434, 2006.

27 Harris RA, Bowker-Kinley MM, Huang B and Wu P: Regulation of the activity of the pyruvate dehydrogenase complex. Adv Enzyme Regul 42: 249-259, 2002.

28 Houten SM, Chegary M, Te Brinke H, Wijnen WJ, Glatz JF, Luiken JJ, Wijburg FA and Wanders RJ: Pyruvate dehydrogenase kinase 4 expression is synergistically induced by AMP-activated protein kinase and fatty acids. Cell Mol Life Sci 66: 1283-1294, 2009.

29 Urruticoechea A, Smith IE and Dowsett M: Proliferation marker Ki-67 in early breast cancer. J Clin Oncol 23: 7212-7220, 2005.

30 Hankinson SE: Circulating levels of sex steroids and prolactin in premenopausal women and risk of breast cancer. Adv Exp Med Biol 617: 161-169, 2008.

31 Ellis BC, Graham LD and Molloy PL: CRNDE, a long noncoding RNA responsive to insulin/IGF signaling, regulates genes involved in central metabolism. Biochim Biophys Acta 1843: 372-386, 2014.

32 Higashi Y, Holder K and Delafontaine P: Thiazolidinediones upregulate insulin-like growth factor-1 receptor via a peroxisome proliferator-activated receptor $\gamma$-independent pathway. J Biol Chem 285: 36361-36368, 2010.

33 Greiner EF, Guppy M and Brand K: Glucose is essential for proliferation and the glycolytic enzyme induction that provokes a transition to glycolytic energy production. J Biol Chem 269: 31484-31490, 1994.

34 Slade N, Storga-Tomic D, Birkmayer GD, Pavelic K and Pavelic $\mathrm{J}$ : Effect of extracellular NADH on human tumor cell proliferation. Anticancer Res 19: 5355-5360, 1999.

35 Pelzmann B, Hallström S, Schaffer P, Lang P, Nadlinger K, Birkmayer GD, Vrecko K, Reibnegger G and Koidl B: NADH supplementation decreases pinacidil-primed IK (ATP) in ventricular cardiomyocytes by increasing intracellular ATP. Br J Pharmacol 139: 749-754, 2003.

$36 \mathrm{Lu} \mathrm{H}$, Burns D, Garnier P, Wei G, Zhu K and Ying W: P2X7 receptors mediate NADH transport across the plasma membranes of astrocytes. Biochem Biophys Res Commun 362: 946-950, 2007.
Received September 8, 2016

Revised September 21, 2016

Accepted September 23, 2016 